\title{
ETAPAS DEL DESARROLLO HUMANO
}

María Eugenia Mansilla A.

\section{RESUMEN}

En este artículo se presenta la discusión del concepto del desarrollo humano y sus diferentes etapas, en cuya formulación se han considerado las variables edad. ambiente, problemas así como las consecuencias psicológicas en los seres humanos; la base teórica; el análisis realizado; la bibliografía consultada y de algunas de las investigaciones realizadas, publicados e inéditas de la autora; y, la propuesta "Etapas del Desarrollo Humano".

Palabras Claves: Desarrollo, etapas del desarrollo, niñez, adolescencia, juventud, adultez, "edad dorada", "edad platino", senectud, gerontes.

\begin{abstract}
In this article it is presented the discussion of the concept of the human development and their different stages in whose formulation has been considered the variable age, atmosphere, problems as well as the psychological consequences in the human beings; the theoretical base; the carried out analysis; the consulted bibliographic and of some of the carried out investigations, published and unpublished of the author; and, the proposal Stages of the Human Development."
\end{abstract}

Key words: I develop, stages of the development, childhood, adolescence, youth, adulthood, "golden age," age platinum", senility, gerontes. 


\section{INTRODUCCIÓN}

Desde la perspectiva psicológica, el estudio del desarrollo humano tiene una antigua historia. Este análisis se inició con los aportes de GESELL en cuanto a maduración y crecimiento, de MOSCOVICI con su propuesta de una psicología social tripolar, y de NEWMAN y NEWMAN con su PSICOLOGÍA ECOLÓGICA, revalorizando los aportes de la Gestall Pero si bien estos autores han contribuido desde diversos enfoques al conocimiento del desarrollo humano, no necesariamente sus propuestas estaban dirigidas a explicarlo en su totalidad puesto que, algunos autores van de lo específico a lo general, otros enfocan el tema como totalidad; más aún, los intentos de los intentos de establecer períodos de desarrollo de algunos autores aún no logran el consenso en el ambiente académico. Así, durante el dictado del curso "Problemas Psicológicos del Desarrollo Humano", de la Maestría en Psicología Educativa de la Universidad Nacional Mayor de San Marcos, ha sido necesaria la discusión teórica del concepto "Desarrollo Humano" y las propuestas explicativas del mismo surgiendo el planteamiento que a continuación se presenta.

En cuanto al concepto desarrollo, MAIER (1969) diferencia el crecimiento orgánico de desarrollo del desarrollo "humano" propiamente tal, que lo relaciona con el desarrollo socio-psicológico y lo define como la "integración de los cambios constitucionales $\mathrm{v}$ aprendidos que conforman la personalidad en constante desarrollo de un individuo "; especificando que, "el desarrollo es un proceso." el cambio. es un producto ". GOOD y BROPHY (1996), consideran que desarrollo "es una progresión ordenada o niveles cada vez más altos tantos de diferenciación como de integración de los componentes de un sistema ". CRAIG (1997: p.29) plantea que desarrollo son los "cambios temporales que se operan en la estructura, pensamiento o comportamiento de la persona y que se deben a factores biológicos y ambientales ". Al referirse a los procesos biológicos este autor considera la maduración, el crecimiento hasta alcanzar la madurez y el envejecimiento: en cuanto a lo segundo, se puede interpretar que lo ambiental es lo "cultural", por tanto se refiere a procesos psicológicos puesto que esta última es producto del quehacer del hombre y es la que determina las condiciones y calidad de vida en la que desarrolla el ser humano. Para SULLIVAN (1983:6), el desarrollo es el "producto de la continua interacción entre diversos factores estimulantes y una matriz de crecimiento prevalecientemente compuesta por ciertas predisposiciones selectivas, tanto para experimentar el cambio como para responder al ambiente de determinadas maneras".

Por la sucinta revisión se observa coincidencia en que desarrollo es cambio e involucra procesos; además, los dos últimos autores mencionan el ambiente como un factor importante en esos procesos, al igual que NEWMAN y NEWMAN (1983) y BROFENBRENNER (1980).

En cuanto a propuestas de etapas acerca del desarrollo, tanto CRAIG (1994) como RICE (1977) exponen la propuesta de ERICKSON dividida en dos partes: la primera que comprende las etapas Prenatal, Infancia, Niñez Temprana, Niñez, Adolescencia; y, una segunda producto de cambios históricos: Adultos Jóvenes (20 a 30 años), Edad Madura (40 a SO años). y de 60 a más años la Edad Adulta Posterior: si bien Rice (1997) diferencia tres períodos de Desarrollo: Desarrollo Infantil con cuatro sub-períodos: Prenatal desde la concepción al nacimiento, Infancia (0-2 años), Niñez Temprana (3-5 años), Niñez Intermedia (6 a II años). Tanto estas como otras propuestas, trabajan intensa y detalladamente los primeros años y tratan sin detalles grandes períodos de la vida del ser 
humano que son visiblemente diferenciales, especialmente a partir de la II Guerra Mundial en que los cambios sociales se aceleran paralelos a la producción de los adelantos tecnológicos.

Para efectos del curso citado se ha realizado el análisis de las etapas de desarrollo, observando la interacción de los tres tipos de edades del ser humano: La edad cronológica / genética, la edad social y la edad psicológica, y se reconocen los diferentes grados de interacción y el mayor o menor peso en el cambio por el patrón de desarrollo genético o por lo histórico en cada etapa; y, teniendo presente que cualquiera sea la variable predominante en los procesos de cambio, siempre estarán mutuamente influidos por la variable cultura / ambiente. Por último, Además, para determinar las etapas del desarrollo psicosocial del ser humano, se consideran las características generales que deben tener todos o la mayoría de los miembros del grupo señalado.

\section{LAS ETAPAS DEL DESARROLLO BIO-PSICO-SOCIAL}

Hasta el siglo XIX existían con absoluta claridad solamente dos categorías reconocidas de seres humanos en cuanto a desarrollo: los mayores y los niños que muchas veces se consideraban igual que los mayores sólo que más pequeños físicamente. Es este el primer referente válido a inicios del siglo XX y determina dos gruesos grupos de desarrollo humano: Los Mayores y los Menores de edad, grupos que también tomamos al iniciar el análisis al que incorporamos un período previo: el que va de la concepción al nacimiento.

\section{DE LA CONCEPCIÓN AL NACIMIENTO}

El desarrollo humano antes del nacimiento se encuentra en una discusión entrampada, por un lado con la postura de la Iglesia y por otra con la postura feminista de la libre determinación del uso del cuerpo y a la determinación de la mujer a continuar o suspender el embarazo, a lo que se suma la práctica de millones de mujeres no feministas a aceptar el embarazo sin mayores discusiones o suspenderlo cuando ella y/o su pareja así lo determina. En estos momentos, tomar una decisión frente a este punto, más que un conocimiento científico es una postura filosófica o religiosa la que sustenta los diferentes planteamientos en cuanto al inicio del ser humano en el feto concebido.

El necesario primer punto a discutir es que, desde la concepción el feto es un ser vivo ya que tiene actividad cerebral y procesos de cambios orgánicos. Como tal capta estímulos y como desde que es concebido comparte la vida de su madre su desarrollo será apropiado o sufrirá las consecuencias de las carencias nutricionales y/o afectivas a través de esta, o los efectos de cualquier agresión física o psicológica indirecta o directa a través de la madre, o cuando se interviene en el feto (Cf. CELADA, 1989). Es decir, el feto inicia un desarrollo propio de un ser humano desde la concepción, momento en que recibe su herencia genética y colectiva. y por sus primeras interacciones con la madre y a través de ella con el medio. Por lo que. aún cuando su proceso de socialización esté limitado al desarrollo del sistema nervioso con que cuenta para realizar sus procesos superiores, desde ya inicia su camino para llegar a ser humano. Esto es importante y se relaciona con el tema del libre albedrío y con la ciencia y sus productos tecnológicos y radicales tales como la fecundación in Vitro, el alquiler de vientre, la intervención genética, la potencial clonación de seres humanos y la decisión a seguir o no el embarazo según el sexo u otras características deseadas o no del niño concebido.

Por eso y para efectos de este trabajo, suscribo la propuesta de los autores mencionados, y denomino el primer período de desarrollo humano como "ETAPA 
PRENATAL", que comprende desde la concepción hasta el nacimiento; período en el que empieza a formar, además de su cuerpo, su psiquis como ser humano.

\section{LOS MENORES DE EDAD}

A mediados del siglo XX se logra el consenso científico en cuanto a que el ser humano alcanza su máximo desarrollo a los 18 años en promedio: en lo biológico, logrando la fortaleza física y capacidad reproductora sin riesgos; y, en lo psicológico, adquiriendo las estructuras que permiten el adecuado interactuar dentro del grupo social de los "mayores".

Es decir, teóricamente se puede considerar que existe un punto del desarrollo en que el ser humano logra el pleno desarrollo del potencial humano con que nació; en ese momento, el "mellar" deja de serio para pasar a ser un "mayor", capaz de interactuar madura y responsablemente en el conjunto social. En 1948 las Naciones Unidas reconoce esta separación en dos grupos y denomina oficialmente "NIÑOS" a los menores: y en 1989, por la "Convención de los Derechos del Niño ", los define como todo ser humano desde que nace hasta que alcanza la "mayoría de edad" 2 . Si bien, esta definición dificulta el consenso social global, porque la transición a la mayoría de edad depende del factor cultural "edad", es un primer paso porque, si se dejan de lado los enfoques políticos particulares conque se determina la edad en que se reconocen la mayoría de edad en cada país, se reconoce legalmente durante la década del noventa ${ }^{3}$ un primer grupo poblacional denominado "mayores de edad" y un segundo grupo denominado "niños ".

\subsection{Los Niños}

La Convención (op.cit.) designa con la palabra niño a todo ser humano entre los $\mathrm{O}$ hasta cumplir los 18 años, pero este es un período de acelerados cambios biopsico-sociales. Un punto importante para una primera subdivisión es el proceso de transición que se vi ve entre los 11 a 12 años, determinado por lo biológico y lo psicológico, identificado por la aparición de los caracteres sexuales secundarios y sesgado por la cultura, denominado "pubertad". Por su importancia individual y social, en las culturas con menos exigencias sociales por el tipo de desarrollo cultural que han alcanzado, esta transición ha significado el paso a la vida adulta y se ha celebrado con ceremonias especiales.

En el Perú. al igual que en la mayoría de países, cumplir los 12 años significa el término de los estudios primarios y, en el mejor de los casos, el paso a los estudios secundarios; si bien, para muchos, es el paso a la incorporación temprana a las actividades de trabajo; así, por el "Código de los Niños y Adolescentes" (Ministerio de Justicia, 1993) reconoce la transición señalada, considerando dos sub-períodos: los "Niños" (O a 11 años); y, los "Adolescentes" (12 a 18 años).

La niñez propiamente tal es uno de los períodos de desarrollo humano mejor estudiado y, por tanto, existe ya un consenso en que el diferente ritmo crecimiento observable en pocos años dentro de la población que la conforma, requiere diferentes satisfactores a sus necesidades en relación inversa a su edad, por lo que se puede señalar dos grupos claramente perfilados:

2 La tendencia de los países-miembros de las NN. UU. , es aceptar la edad de 18 años señalada por la Convención.

3 Este reconocimiento legal es importante porque conlleva necesarios cambios en la estructura social y en las políticas sociales que pueden contribuir al desarrollo humano. 
a. La Primera Infancia (0-5 años) caracterizada por un alto grado de dependencia y su alta morbi-morbilidad, características que lo ponen en alto grado de riesgo en relación inversa a la edad, por lo que requiere un estricto "Control de Desarrollo y Crecimiento ". De aquí, en la categoría "niños", el grupo de O a 1 año sea de muy alto riesgo; el grupo de 2 a 3 años de alto riesgo; y, el grupo de 4 a 5 años que tiene mayor atracción a los intereses exogámicos, como de menor riesgo.

Por la condición predominante de riesgo de morbi-mortalidad se le denomina "Edad Vulnerable "; y,

b. La Segunda Infancia (6 a 11), se caracteriza por su apertura al mundo externo y por la acelerada adquisición de habilidades para la interacción. En este período los niños tienen un riesgo menor que en la primera infancia, que disminuye igualmente en razón inversa a la edad, por lo que el Control de Desarrollo y Crecimiento se realiza anualmente.

Por las consecuencias que la falta de satisfactores apropiados a sus necesidades psicosociales produce en este grupo de niños, la denominamos "Edad Crítica" (MANSILLA, 1987, 1990, 1996).

\subsection{Los Adolescentes ${ }^{4}$}

"Adolescente" es un término recientemente adoptado en diversos países de habla española y es coincidente con el término "teenagers" (13- 19 años) de USA, y el Código de los Niños y Adolescentes (M. Justicia, 1993) reconoce como tales a la población desde los 12 a los 17 años.

En este período de desarrollo se vive un crecimiento acelerado del esqueleto provocando cambios en la autoimagen y en el manejo físico del espacio, especialmente hasta los 14 años, todo lo cual dificulta el manejo de las interacciones; además, se terminan de desarrollar los sistemas respiratorio, circulatorio y de la reproducción. En cuanto a lo psicológico aparece una inquietud dirigida a explorarse a sí mismo y el entorno; asimismo, en este período definen su identidad social y de género. Una característica psicológica importante y creativa es que, alrededor de los 15 años empiezan a creer que pueden cambiar y dominar el mundo, lo que puede desbordarlos, y en el caso de la adolescente frustrarla por razones de género si el medio que los rodea es estrecho y sin adecuados satisfactores sociales a sus necesidades.

Relacionando los factores que hemos señalado, podemos establecer que existen diferencias notables entre los adolescentes de 12 a 14 años $^{5}$ y los de 15 a 17 años, que se agudizan en el caso de un importante sector de la población que alrededor de los 14-15 años empieza a incursionar en las actividades laborales lo que se acompaña de la exploración activa de la sexualidad y deriva en la búsqueda de pareja; si bien, salvo excepciones, aún no tienen la estabilidad emocional, las habilidades laborales y sociales, y

\footnotetext{
${ }^{4}$ Se debe recordar que recién en el siglo XIX se han reconocido y aceptado socialmente las diferencias de las que tratamos en este artículo, que permiten establecer las categorías basadas en la esperanza de vida y mejora de la calidad de vida actuales.,

${ }^{5}$ Hasta hace poco en muchos países, y hasta el presente en algunos pocos, a esta edad se aceptaba el matrimonio y la procreación
} 
el manejo de la información necesaria para asumir dichas actividades y más bien cuentan con y exigen el apoyo familiar, especialmente los que aún son estudiantes.

Estas características y las diferencias observables en el grado de desarrollo, llevan a reconocer dos sub-períodos dentro de la categoría "adolescencia": los "adolescentes primarios ", individuos de 12 a 14 años; y, los "adolescentes tardíos ", individuos de 15 a 17 años. Para este grupo, se produce un vacío social en las respuestas sociales favorables a su desarrollo (muchos sin posibilidad de estudiar y buscando trabajo) y más bien se le considera plausible de imputabilidad legal por actos de terrorismo y de "pandillaje pernicioso ". Este entorno lo hemos denominado "limbo social".

Al finalizar la "adolescencia tardía" se vive un período de transición entre la adolescencia y la mayoría de edad, que tiene una importancia incuestionable.

En resumen, por las características de este segundo período de Desarrollo Humano, de formación, crecimiento y desarrollo, lo denominamos ETAPA FORMATIVA.

\section{LOS MAYORES DE EDAD}

Al llegar a los 18 años los individuos de ambos sexos, en la mayoría de países, son incorporados formalmente, sin mayores ceremonias a la sociedad civil mediante algún tipo de identificación oficial ${ }^{6}$ que les reconoce como "mayores de edad" hasta el fin de su vida y les permite:

- Responder por sus actos ante la justicia;

- Participar en el sistema de defensa nacional ${ }^{7}$, y por tanto matar y morir en acciones armadas;

- Participar en la Población Económicamente Activa, PEA, a excepción de los que siguen estudios profesionales y técnicos.

- Participar en las actividades sociales y políticas; y,

- Unirse en matrimonio y formar familia

Para asumir estos deberes y derechos, los "mayores de edad" deben haber logrado durante la Etapa Formativa, entre otras características, las habilidades y destrezas para desenvolverse con autonomía de pensamiento y acción en la vida social y, para adquirir compromisos de familia propia y relaciones de pareja. Son estas dos últimas características las que, con variaciones, acompañan a los individuos hasta el fin de su vida.

Los mayores de edad de ambos sexos, con diferencias en los procesos, tienen un período de transición que conocemos como "Climaterio ", al mismo tiempo que una característica social que produce una diferencia significativa entre sí, que los divide en dos grupos: los que trabajan (o buscan un trabajo) y los que dejan de trabajar (o debieran dejar de hacerla). Por ambas importantes características podemos dividirlos en dos etapas diferenciadas: los "Trabajadores" y los "Jubilados ".

\footnotetext{
${ }^{6}$ En el Perú el documento de identidad nacional DNI.

${ }^{7}$ En algunos países esta incorporación al sistema de defensa es a los 16 años.
} 


\subsection{Los Trabajadores}

A diferencia de la creatividad que nos acompaña en diversos grados desde que nacemos hasta que morimos, la actividad laboral varía en calidad y cantidad según sea la edad del trabajador. Los "Trabajadores" per se son la fuerza activa de la producción económica (Población Económicamente Activa - PEA). Si bien en el Perú se ha legislado la incorporación al trabajo de adolescentes (14 a 17 años) y pueden permanecer activos los mayores de 65 años en actividades de trabajo. la PEA formal es de 18 a 65 años, edades que serán los límites de este período.

Los "Trabajadores" pueden mantenerse y mantener o contribuir significativamente a mantener a terceros con el producto de su trabajo: pueden formar familia: y pueden participar activamente en la vida social del país a través de actividades políticas y comunitarias. Al finalizar este período los seres humanos viven un tercer proceso de transición determinado por lo biológico y sesgado por la cultura: el Climaterio, que deja ver las consecuencias de los estereotipos sexuales en cada individuo, a lo que se suma que al cumplir los 6S años deben dejar de trabajar pasando a jubilarse.

Como el período señalado es aún muy grande (18 a 65 años), se observan diferencias psico-sociales importantes que llaman a divididos, en primer lugar, entre los Jóvenes y los Adultos.

\subsection{Los Jóvenes}

UNESCO, al igual que Estados Unidos de Norteamérica, consideran los 14 o más años de la adolescencia con los primeros años de la madurez en la categoría "Jóvenes"; sin embargo las diferencias entre los adolescentes tardíos y los individuos que pasan a ser mayores son sustanciales como ya se explicó en párrafos anteriores.

Siguiendo la separación señalada, los seres humanos que se encuentran entre los 18 y 24 años de edad son maduros en cuanto crecimiento y desarrollo psicológico, pero son diferentes a los demás mayores por ser inexpertos en el mundo adulto, al que entran con entusiasmo, dinamismo y creatividad que la compensan; y, mantienen aún una gran facilidad para el cambio lo que facilita el aprendizaje y aprehensión de conocimientos e información a velocidad, todo lo cual se expresa en su activa y muchas veces creativa participación social. Al finalizar el período, ya tienen estudios terminadas (ha excepción de las carreras profesionales muy largas). tienen trabajo y tienen o han tenido relaciones de pareja: pocos se han casado. los más tienen en miras adquirir responsabilidades de familia propia. Actualmente se observa, especialmente en los jóvenes con estudios superiores, caracteres psicológicos de competencia y de logro altísimos y un distanciamiento de proyectos de familia propia. En cuanto a los estereotipos sexuales, presentan un nuevo modelo de relaciones de pareja y forma de ver el "mundo", que se les presenta más amplio y más exigente.

Para nuestros objetivos, y por las características ya señaladas, denominamos "Jóvenes" a todos los seres humanos desde los 18 a los 24 años. 


\subsection{Los adultos}

Adulto es todo ser humano desde los 25 a los 64 años. En este sub-período, además de los cambios biológicos, las diferencias en el desarrollo se relacionan con la mejor calidad de vida y la mayor esperanza de vida, lo que influye en la personalidad de sus integrantes y en su quehacer social; $y$, se contemplan en tres subgrupos:

a. Adultos Jóvenes o adultos primarios (25 a 39 años). Ya cuentan con un trabajo y una familia en crecimiento que, especialmente al inicio de la categoría, les produce conflictos por su tendencia a la actividad juvenil frente a las responsabilidades familiares y laborales. Es decir, enfrenta la madurez de su desarrollo. Al llegar al final de esta sub-categoría se observan cambios físicos, tales como canas, arrugas iniciales, pérdida de flexibilidad principalmente por falta de ejercicio, y se alcanza un punto importante del desarrollo intelectual. Pese a tal. como el Perú tiene un modelo de producción económica que prioriza la competencia por sobre la competitividad. se presenta un gran problema con graves consecuencias psico-sociales: un significativo porcentaje de la población urbana ha perdido su trabajo y se encuentra sub-empleada o está desempleada.

b. Adultos o adultos intermedios (40 a 49 años). En este grupo predomina el enfrentamiento a cambios familiares y entre parejas, producido en parte -o quizás el detonante - por el proceso de cambio de los estereotipos sexuales que está dando paso a nuevas formas de interacción entre sexos y entre generaciones, especialmente si tuvieron a sus hijos a temprana edad: Además se enfrentan a notables cambios derivados del Climaterio que, hoy se ha comprobado, acune con poca diferencia de edad en ambos sexos pero es más dramático, por ser visible, en las mujeres que ya entran a la Pre-menopausia. Las canas, la pérdida de cabello y la flacidez muscular, especialmente en el hombre, son considerados negativos porque se contraponen con el actual modelo de "juventud" perenne, especialmente en el campo laboral donde se ven día a día desplazados. De todo esto derivan problemas psico-sociales novedosos y serios si no hay prontas soluciones.

c. c. Adultos mayores o adultos tardíos (50 a 64 años). En este grupo, además del proceso biológico del Climaterio que llega a su final con todas sus consecuencias psicológicas, especialmente en la mujer, los individuos deben enfrentar el alejamiento de los hijos (síndrome del nido vacío) ya iniciado en la década anterior, sea por estudios o por la formación de nuevas familias. pero que no llegan a irse del todo por las condiciones económicas. Al finalizar este período. la mayoría enfrenta a la posibilidad de dejar de trabajar. jubilarse. lo que conlleva dos problemas diferentes pero complementarios: qué hacer con el tiempo disponible y la capacidad productiva aún existente, y cómo solventar actividades y la economía del hogar.

Al largo e importante período de la vida del ser humano comprendido desde los 18 hasta cumplir los 64 años, por su característica principal: la capacidad laboral y de inserción en el trabajo, lo denominamos ETAPA LABORAL. 


\section{LOS JUBILADOS}

Cuando el trabajador cumple 65 años puede proceder a jubilarse. Jubilado significa que el trabajador ha dejado de ser tal para pasar a retiro de la actividad económica; y, jubilar es la acción de eximir de la actividad laboral por edad, al mismo tiempo que significa, entre otras acepciones, alegrarse. Por tanto, "jubilarse" debiera ser el periodo de vida en que el ser humano recibe (o debiera recibir) "alegremente" los beneficios acumulados durante su etapa laboral: pensión, rentas, familia extendida que le puede prestar soporte afectivo, etc., lo cual aún no es una realidad.

El estudio de esta población es reciente, cuenta con pocas décadas, pero se pueden observan cambios en el desarrollo humano que permiten señalar dos grupos: los Senectos, y los Gerontes.

\subsection{Los "Senectos"}

Los senectos son los adultos de 65 a 74 años. Este término es sinónimo de Vejez. por tanto significa ancianidad: sin embargo el término senecto se confunde con senilidad, término que es una categoría psiquiátrica y designa una enfermedad mental. Lo importante es que hoy se discute cuando se es anciano y se diferencia el proceso por el cual se envejece de las diferentes enfermedades que pueden atacar al ser humano. Existen una serie de prejuicios acerca de los envejescentes en cuanto son pasivos, son asexuados, no puede seguir aprendiendo, que tienen una actitud de indiferencia y abandono, etc. NEUGARTE (1970) considera que el término senectismo describe el prejuicio de lo que es ser anciano, igualmente el "viejísimo". Pero, ¿quién puede informar científicamente que este grupo humano tiene mayoritariamente tal actitud? $\mathrm{O}$ que, ¿esta actitud no es aprendida socialmente?

Los senectos son individuos que tienen experiencia dentro del campo laboral de cada uno, muchos han sobrevivido a guerras, otros a hambruna, otros a desastres naturales, y la mayoría ha sacado adelante una familia. Es decir, son personas valiosas socialmente pero requieren nuevos entornas y nuevas repuestas sociales a sus necesidades. ¿Cuánto de los prejuicios no es resultado de la ausencia de respuestas y entornas apropiados a sus necesidades?

Las diferencias encontradas dentro de este sub-período lleva a dividirlo en dos subgrupos: los senectos primarios (65 a 69) y los senectos intermedios (70 a 74 años). Es durante el primero que se produce la confrontación con el sí mismo y la vida. Al pasar al segundo va encontrando paz para vivir consigo mismo y el mundo. si bien ya pierde en la mayoría de los casos a su pareja y, en los países en desarrollo con débiles programas de jubilación, pasa a ser dependiente de sus descendientes.

EsSalud, Empresa de Bienestar Social, ha organizado el Programa Edad Dorada para todos los Jubilados. Por extensión y solo para los miembros de esta categoría denominamos este sub-período EDAD DORADA. 


\subsubsection{Los Gerontes}

Considerando una disposición social, que empieza a tener vigencia, determina que al cumplir los 75 años deben dejarse vacantes los cargos sociales por ejemplo, la jerarquía eclesiástica-, razón por la cual hemos reconocido la división de un nuevo sub-grupo: Los "gerontes" que son los ancianos mayores de 75 años. El actual modelo social no favorece el reconocimiento de la experiencia y otras habilidades individuales que se logran con el paso de los años y ya pasó la época en que los Consejos de Ancianos eran fundamentales para la transmisión oral y para el consejo a la comunidad. Hoy algunos ejercen cargos similares y si bien son excepcionales, en Perú tenemos algunos ejemplos de Gerontes, tanto vivos como muertos:

Basadre (historiador), Belaúnde (Estadista), Jorge del Prado (sindicalista), Luis Alberto Sánchez (Literato), etc. Y la lista internacional es demasiado larga y conocida. ¿Por qué se acepta sólo las excepciones y no a la mayoría? ¿Por qué no se reconoce la vejez como un paso más en el continuo del desarrollo humano que a todos. más tarde o temprano nos tocará recorrer? Principalmente porque existe miedo al deterioro físico en que caen algunos ancianos y temor a la muerte.

Los Gerontes se pueden dividir en:

Senectos Tardíos (75 a 84 años); Longevos (85 a 94 años); y, Prolongevos (95 a más años), término acuñado por Gerald J. GRUMAN para designar "los esfuerzos deliberados para extender la vida por medio de la acción humana" (RICE, op.cit. p: 521), y que hacemos extensivos a todos los mayores de 95 años, vivan una prolongado vida natural o con intervención de la ciencia.

Por lo extraordinario de llegar a vivir este último período la vida lo denominamos EDAD PLATINO Y el período que comprende la "Edad Dorada" y la "Edad Platino", la denominamos ETAPA JUBILAR.

Esta breve descripción de etapas y sub-etapas nos lleva a una pregunta medular: ¿Cuánto es producto de la sociedad por sobre lo biológico? Porque al investigar sobre el desarrollo humano se comprueba la diferencia entre la percepción de la vejez y la autopercepción de los ancianos de 15 o 20 años atrás y los de hoy.

Esto nos lleva a la recomendación de investigaciones más finas para delimitar las etapas de desarrollo en relación al actual desarrollo social que influye en el desarrollo integral de los seres humanos tanto positiva como negativamente.

A continuación presentamos una propuesta de las etapas biopsico-sociales del desarrollo humano. 
ETAPAS BIO-PSICO-SOCIALES DEL DESARROLLO HUMANO

\begin{tabular}{|c|c|}
\hline ETAPAS & SUB- ETAPAS \\
\hline I. Etapa Prenatal & Desde la gestación del nacimiento \\
\hline $\begin{array}{l}\text { II. Etapa Formativa } \\
\text { 0 a } 17 \text { años }\end{array}$ & $\begin{array}{l}\text { 2.1 Niñez (0 a } 11 \text { años) } \\
\text { - Primera infancia (0 a } 5 \text { años) } \\
\text { - Segunda Infancia (6 a } 11 \text { años) } \\
\text { Transición: Pubertad } \\
\text { 2.2 Adolescencia (12 a } 17 \text { años) } \\
\text { Transición: a la vida laboral }\end{array}$ \\
\hline $\begin{array}{l}\text { III. Etapa laboral } \\
18 \text { a } 64 \text { años }\end{array}$ & $\begin{array}{l}\text { 3.1 Juventud (18 a } 24 \text { años) } \\
\text { 3.2 Adultez (25 a } 64 \text { años) } \\
\text { - Adultos jovenes (25 a } 40 \text { años) } \\
\text { - Adultos intermedios (41 a } 50 \text { años) } \\
\text { - Adultos mayores (51 a 64) } \\
\text { Transición: Climaterio femenino y } \\
\text { masculino }\end{array}$ \\
\hline $\begin{array}{l}\text { IV. Etapa jubilar } \\
65 \text { a }+ \text { años }\end{array}$ & $\begin{array}{l}\text { 4.1 Etapa Dorada } \\
\text { - } \quad \text { Senectos primarios }(65 \mathrm{a}+69) \\
\text { - } \quad \text { Senectos intermedios }(70 \text { a } 74) \\
\text { 4.2 Etapa Platino } \\
\text { - } \quad \text { Ancianos }(75 \text { a } 84) \\
\text { - } \quad \text { Longevos }(85 \text { a } 94) \\
-\quad \text { Prolongevos }(95 \text { a + años })\end{array}$ \\
\hline
\end{tabular}




\section{BIBLIOGRAFÍA}

Ausubel, O.P., Sullivan, E.V. (1983), El desarrollo infantil. Barcelona, Paidos, Biehler, R.F. (1980), Introducción al desarrollo del niño. México, Editorial Diana

Brofenbrenner, (1979), The ecology of human development. Cambridge: Harvard University Press Celada G., J. (1989), Desórdenes Psíquicos. Lima:Serie N M]Neuropsicología y Rehabilitación, Vol. 1, p. 20.

Clemente, A. (1996), Psicología del desarrollo adulto. Madrid, Ed. Narcea S.A.

Craig, G, J. (1994), Desarrollo Psicológico. México: Prentice Hall Hispanoamericana, S.A.

Davis, M., Gonzales, M., Envejecer con alegría. Un desafío educacional. En:

Encuentro de Innovadores e Investigadores en Educación. Mar del Plata.

Hoffman, L., Paris, S., Hall, E. (1996), Psicología del desarrollo hoy, Madrid, Ed. McGraw-Hill

Liado B., M. (1990), Geriatría. Vejez y envejecimiento: sus problemas. Lima, Universidad Inca Garcilaso de la Vega.

Maier, H. (1968), Tres Teorías sobre el desarrollo del niño: Erickson, Piaget y Sears. BS.As: Amorrortu Editores.

Mansilla A., M.E. (2000), Problemas del Desarrollo Humano, en preparación.

Mansilla A .. M.E (1999). La socialización en la calle. En: Revista de Psicología. Lima. Universidad Nacional Mayor de San Marcos. Año 111. ํ 4. Ene 99.

Mansilla A .. M.E (1998). Realización de Necesidades y Desarrollo Humano. En:

Anales de Salud Mental. Lima. Instituto Nacional de Salud Mental "Honorio Delgado - Hideyo Noguchi. Vol XIV.Ns. 1 y 2.

Mansilla A., M.E (1996), La Socialización Diferenciada por Sexo. Lima: CONCYTEC, Serie Socio Cultural $\mathrm{N}^{\circ} 001$.

Mansilla A., M.E (1988), El Desarrollo Psico-social del niño y adolescente. Lima. UNICEF. Módulo de Capacitación.

Mansilla A., M.E (1977), Los niños y adolescentes en situación de riesgo. Lima, Pontificia Universidad Católica del Perú, ts. para optar al Grado de Magister.

Ministerio de Justicia, (1993), Código del Niño y Adolescente. Lima, Ministerio de Justicia

Naciones Unidas (1948), "Declaración de los Derechos del Niño"

Naciones Unidas (1989), La Convención de los Derechos del Niño. N.Y: UNICEF

Newman, B. y Newman, Ph. (1983), Desarrollo del Niño. México: Ed. LIMUSA

Papalia, D. (1997), Desarrollo Humano. México, McGraw-Hill

Perez, J. (1994), Relación entre individuo y sociedad. En: J.F. Morales, Coordinador: Psicología Social, Madrid, Editorial McGraw-Hill

Pollock. L. (1990), Los niños olvidados. Relaciones entre padres e hijos de 1500 a 1900. México: Fondo de Cultura Económica.

Rice, F. Philip (1997), Desarrollo Humano. México. Prentice Hall Hispanoamericana S.A.

Thorne, C. ( 1994). Nuevas interpretaciones de la psicología del desarrollo con relación a los determinantes de la conducta. En: Psicología. Vol XII, No. 1. Lima. PUCP. 